\title{
SISTEMA INFORMÁTICO PARA EL ANÁLISIS DE LAS PERCEPCIONES SOCIOAMBIENTALES.
}

Ing. Adianys Gil Montero ${ }^{1}$, MSc. Amaury Machín Armenteros ${ }^{2}$, Dr.C Yasser Vázquez Alfonso ${ }^{3}$

M.Sc Efraín Velasteguí López ${ }^{4}$

\begin{abstract}
In the measure in that the concern grows to maintain and to improve the quality of the environment and to protect the human health, diverse institutions are focused in the development of applications that they satisfy this necessities. Being one of these institutions AMA (Agency of the Environment) together with the different dependences of the CITMA (Science, Innovation, Technology and Environment), where the objective of this investigation was to develop a computer system for the analysis of the partner-environmental perception of the earth Slips. You was carried out a study on the different dimensions that it contemplates the partnerenvironmental perception, being defined the technologies and methodologies to use together with the mathematical and computational algorithms, the software SIAPSADT1.0 was elaborated that allowed to analyze in an automatic way the information. This investigation is of great importance to facilitate the process of taking of decisions on the part of the directive of AMA and of provincial CITMA.
\end{abstract}

Keywords: Environmental Partner Perceptions, SIAPSADT 1.0, Computer System, Earth Slips.

CÓDIGO UNESCO: Sistemas de Información 120318

\section{RESUMEN}

En la medida en que crece la preocupación por mantener y mejorar la calidad del medio ambiente y proteger la salud humana, diversas instituciones se enfocan en el desarrollo de aplicaciones que satisfagan dichas necesidades. Siendo una de estas instituciones AMA (Agencia del Medio Ambiente) en conjunto con las diferentes dependencias del CITMA (Ciencia, Innovación, Tecnología y Medio Ambiente), donde el objetivo de esta investigación fue desarrollar un sistema informático para el análisis de la percepción socio-ambiental de los Deslizamientos de tierra. Se realizó un estudio sobre las diferentes dimensiones que contempla la percepción socio-ambiental, definiéndose las tecnologías y metodologías a utilizar en conjunto

\footnotetext{
${ }^{1}$ Universidad Agraria de la Habana, La Haba, Cuba, adianysg@unah.edu.cu

${ }^{2}$ Universidad de la Habana, La Haba, Cuba, amaurym@unah.edu.cu

${ }^{3}$ Universidad de la Habana, La Haba, Cuba, yalfos1@gmail.com

${ }^{4}$ Universidad Técnica de Ambato, Ambato, Ecuador, le.velastegui@uta.edu.ec
} 
con los algoritmos matemáticos y computacionales, se elaboró el software SIAPSADT 1.0 que permitió analizar de forma automática la información. Esta investigación resulta de gran importancia para facilitar el proceso de toma de decisiones por parte de los directivos de AMA y de los CITMA provinciales.

Palabras Claves: percepciones socio ambiental, SIAPSADT 1.0, sistema informático, Deslizamientos de tierra,

\section{INTRODUCCIÓN}

Los nuevos enfoques en el tratamiento de los peligros de origen natural están incorporando, cada vez más, la inclusión de la dimensión social como un componente que explica en muchos casos la dimensión y magnitud de los impactos que estos peligros generan y por tanto las perspectivas multidisciplinarias e integradoras en la investigación y gestión de riesgos asociados a estos fenómenos. La frecuencia de aparición de estos peligros en nuestras condiciones, demanda de las ciencias en el orden teórico y práctico, resultados que ayuden a elaborar de manera científicamente fundamentada, políticas, estrategias, planes y maneras de actuar para prevenir y mitigar los efectos negativos de estas amenazas en las diferentes escalas de la sociedad: global, territorial y local(Calleja, 2013).

Según Calleja (2013) plantea que uno de los retos para acometer esta tarea radica en la comprensión por parte de todos los actores sociales de que, el conocimiento sobre la gestión de riesgos ambientales no debe ser abordado, como hasta ahora, de manera fragmentada, con énfasis en los aspectos puramente físicos y naturales de los desastres. Lo más frecuente ha sido el énfasis en los datos meteorológicos, hidrológicos y geológicos a la hora de tomar decisiones sobre la prevención y mitigación de estos eventos, prácticamente se ha excluido la información referida a la vulnerabilidad social. En este camino, la contribución de las ciencias sociales ha sido la elaboración de herramientas para el estudio de la percepción en la población del peligro en cuestión.

La percepción, no sólo es un fenómeno sensorial, sino también cultural. A partir de la percepción, se forma el saber geográfico, el cual es la base para la formación de actitudes, posturas y posicionamientos culturales que tomamos frente al mundo (Rodríguez, 2004).

En el mundo de manera general diversas instituciones se enfocan en la realización de sistemas informáticos para analizar y almacenar el funcionamiento de los componentes del medio ambiente entre los cuales se destacan: ISOTools (2013) como plataforma tecnológica para la gestión ambiental. Herramienta informática que integra ACV (Análisis de Ciclo de Vida) y SIG (Sistemas de Información Geográfica) para la evaluación ambiental de sistemas de gestión de residuos en España(Martínez, 2009). 
Cuba no se ha quedado atrás en la investigación e implementación de software para el control y procesamiento de los indicadores del desempeño ambiental uno de ellos es: Sistema de Indicadores para la Evaluación de la Aplicación del Sistema de Gestión Ambiental en Empresas Constructoras en Holguín (Guttman et al., 2004).

Sistema de Evaluación de Impacto Ambiental, software destinado a técnicos especializados y estudiantes, para realizar un estudio del impacto que ocasionará la puesta en marcha de un proyecto, obra o actividad sobre el medio ambiente. A partir de este estudio se predice y evalúa las consecuencias que la ejecución de dichas actividades pueda ocasionar en el entorno en el que se localiza (Mínguez et.al, 2009).

El proceso de evaluar y analizar la encuesta realizada actualmente sobre la percepción socio-ambiental dentro de la Agencia del Medio Ambiente se archiva de forma manual por lo que atenta contra la eficiencia de la interpretación de los mismos, hay pérdida de información cuando se busca algún dato, por lo que se torna engorroso y complejo. A pesar de que existen diferentes paquetes estadísticos como el Data Entry, se considera que no es un software que garantiza toda la confiabilidad en sus resultados, es por eso que se considera que no existe hasta el momento dentro de esta institución ningún software que permita gestionar, procesar y analizar el cuestionario sobre Deslizamientos de tierra.

Esto conlleva a la necesidad de desarrollar un sistema informático que permita gestionar de forma automática la información relacionada con la percepción socio-ambiental a su vez el procesamiento de sus resultados.

\section{Materiales y métodos}

La presente investigación se realizó en el primer semestre del año 2017 en la Agencia de Medio Ambiente (AMA) que se encuentra ubicada en el municipio Playa de la Provincia La Habana.

La metodología de desarrollo de software utilizada para desarrollar este sistema informático fue Proceso Unificado Racional (RUP, por sus siglas en inglés). Este es un proceso de desarrollo de software que se adapta según las necesidades de los desarrolladores y del cliente, debido a que la comunicación entre estos debe ser fluida, permitiendo llegar a un acuerdo para evitar desavenencias futuras, además integra el Lenguaje Unificado de Modelado (UML) y está basado en componentes interconectados a través de una interfaz bien definida.

RUP se caracteriza por ser dirigido por Casos de Uso, centrado en la arquitectura, iterativo e incremental. Posibilita ordenar las actividades de un equipo y dirige las tareas de cada desarrollador por separado y del equipo como un todo. Especifica los 
artefactos que deben desarrollarse, ofrece criterios para el control y medición de los productos (Carrasco, 2015).

Para la elaboración del sistema informático se utilizaron diferentes tecnologías como son: Xampp como servidor, Java como lenguaje de programación, MySQL como sistema de Gestión de Base datos, Visual Paradimg 8.0 como herramienta case, php como lenguaje de programación web y la librería html2fpdf para la generación de reportes (Gil and Lara; 2016).

En el análisis de la información relacionada con la percepción socio ambiental sobre los deslizamientos de tierra se aplicaron diferentes algoritmos de minería de datos como son: ID3 que permitió clasificar el conjunto de instancias ubicada en la base de datos, Apriori extrajo un conjunto de reglas de asociación de la información contenida en la base de datos y el Clúster clasificó la similitud entre los diferentes atributos de la base de datos.

Para la implementación de los algoritmos ID3 y Apriori en el sistema informático se utilizó los pseudocódigos planteados por Hernández (2005), estos son:

ALGORITMO ID3 Partición(N: nodo, E: conjunto de ejemplos)

Si todos los ejemplos E son de la misma clase c ENTONCES Asignar la clase c al nodo N.

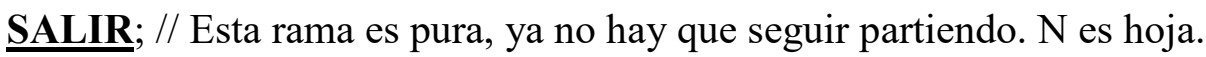

\section{SI NO:}

Particiones:=generar posibles particiones.

MejorPartición:= seleccionar la mejor partición según el criterio de partición.

PARA CADA condición i de la partición elegida.

Añadir un nodo hijo i a $\mathrm{N}$ y asignar los ejemplos consistentes a cada hijo (E).

Partición (i, E). // Realizar el mismo procedimiento global con cada hijo.

\section{FIN PARA}

\section{FIN SI}

\section{FIN ALGORITMO}

Para clasificar un conjunto de ejemplos E, se invoca con la llamada Partición(R,E), donde $\mathrm{R}$ es un nodo raíz de un árbol por empezar.

ALGORITMO Apriori (D:datos, MinC: cobertura mínima)

$\mathrm{i}=0$

Rellena_Item $\left(\mathrm{C}_{\mathrm{i}}\right)$ //Incluye en $\mathrm{C}_{0}$ todos los ítems de tamaño1

MIENTRAS $C_{i} \neq \Phi$

PARA CADA $\mathrm{x}=$ elemento de $\mathrm{C}_{\mathrm{i}}$

SI Cobertura $(\mathrm{x}) \geq \operatorname{MinC} \underline{\text { ENTONCES }} \mathrm{L}_{\mathrm{i}}=\mathrm{L}_{\mathrm{i}} \cup \mathrm{x}$ 
FIN PARA

$\mathrm{C}_{\mathrm{i}+1}=$ Selecciona_Candidatos $\left(\mathrm{L}_{\mathrm{i}}\right)$

$\mathrm{i}=\mathrm{i}+1$;

FIN MIENTRAS

RETORNA C

FIN ALGORITMO

En la implementación del algoritmo de Clúster en el sistema informático se utilizó el pseudocódigo planteado por Garreet et.al. (2007) que consiste en:

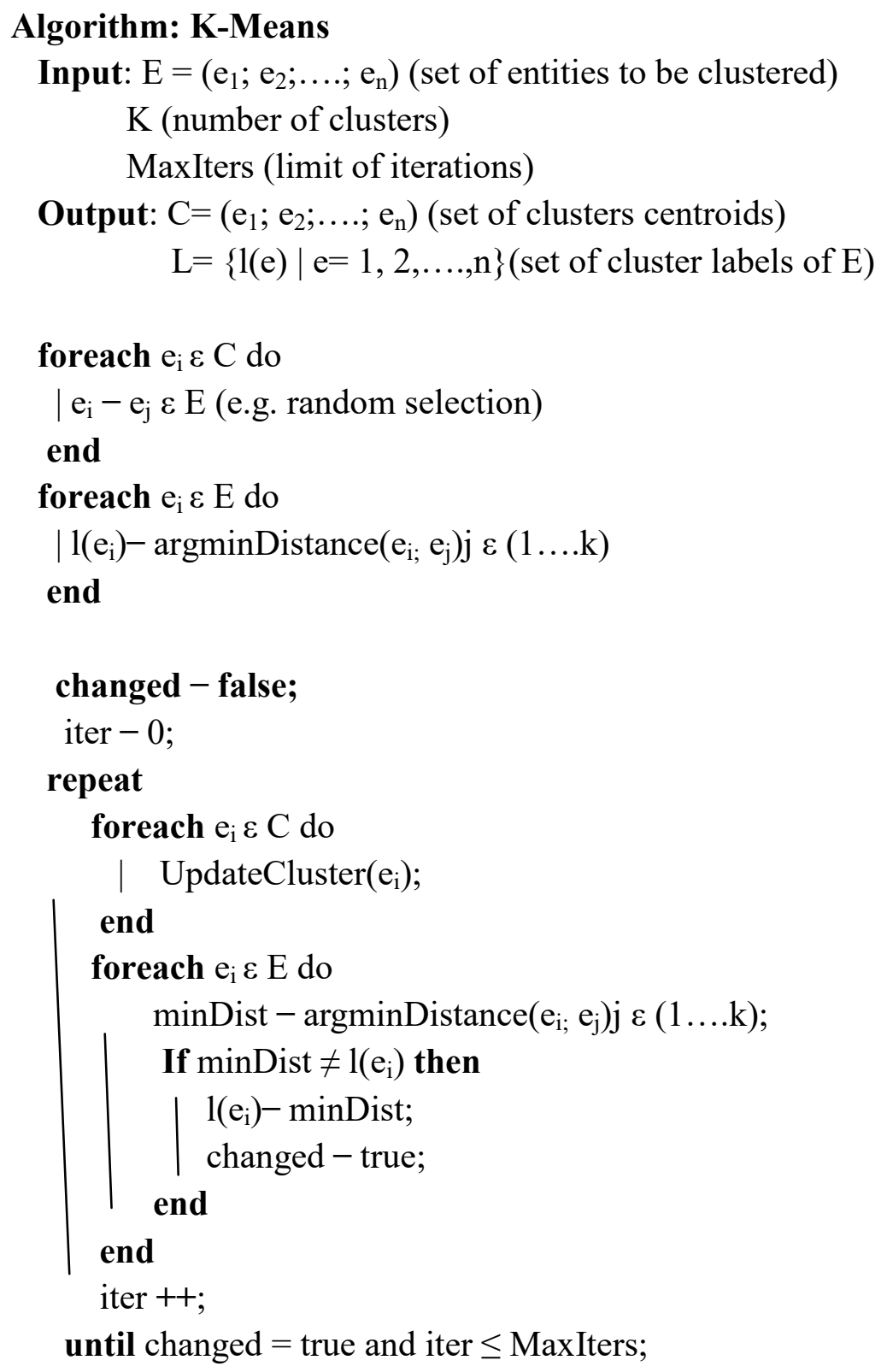




\section{Resultados y discusión.}

El sistema se implementó en ambiente web sobre el servidor Xampp. La primera interfaz que muestra el sistema es la página inicial con el menú para escoger las opciones a realizar según el rol del usuario.

Las ventajas que aporta la implementación de este sistema según los autores de esta investigación son:

- Facilita la recogida y validación de la información relacionada con la percepción socioambiental y mejora de la calidad del dato, al eliminar el tratamiento manual de la información.

- Permite mantener una alta disponibilidad de la información, homogénea y actualizada, lo que ahorra tiempo y recursos a la hora de analizar las métricas del negocio.

- Posibilita el control y comunicación: La información agregada es traducida a lenguaje de negocio y se hace visible y accesible a todos los miembros de la organización. Obviamente, cada usuario tendrá acceso y visibilidad únicamente a la información que necesita.

- Facilita la toma de decisiones de las percepciones socio-ambientales en la Agencia del Medio Ambiente junto con los diferentes CITMA, presenta iteraciones con dos usuarios, el Especialista del CITMA y el Administrador del sistema.

A continuación se muestra la interfaz de bienvenida al sistema SIAPSADT 1.0 (figura 1), en el mismo se muestra imágines sobre el deslizamiento de tierra y una breve descripción del sistema. Esta aplicación le permitirá al usuario según los roles establecido desempeñar sus actividades.

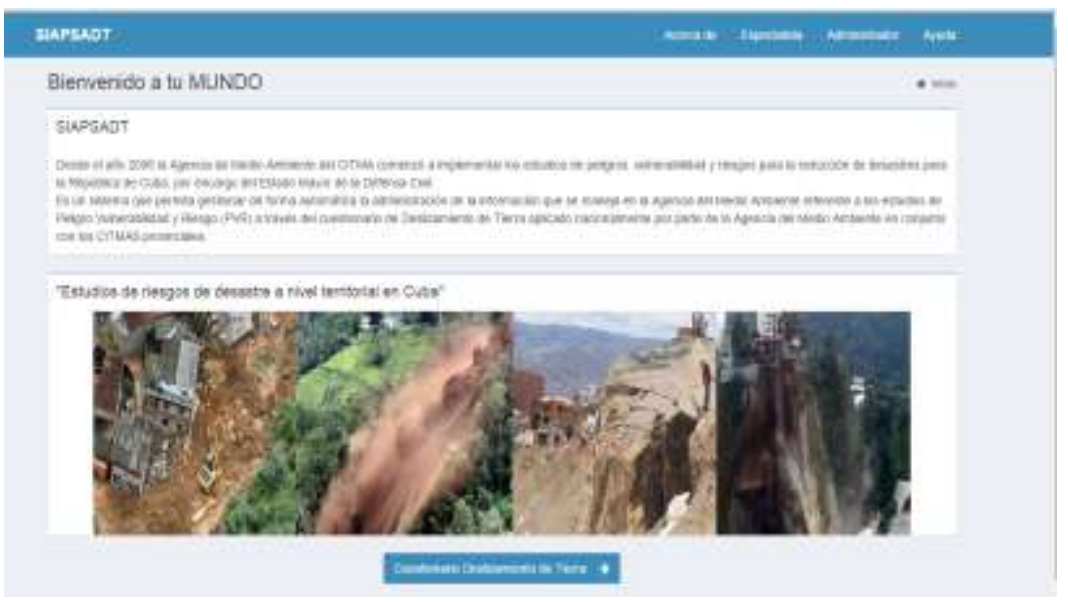

Figura 1: Interfaz de la página principal del sistema.

Fuente: Elaboración propia. 
La figura 2 muestra la interfaz relacionada con usuario Especialista, la misma le permite realizar la encuesta que cuenta con un conjunto de preguntas que se clasificadas en preguntas de selección simples, selección múltiple y de redacción, además de realizar los análisis de la información referente a las percepciones socio-ambientales mediante la aplicación de los algoritmos de minería de datos: Árboles de decisión (ID3), Reglas de asociación (Apriori) y Cluster (SimpleK-Means), así como las gestiones con los resultados obtenidos por las encuestas aplicadas a través de gráficos 2D.

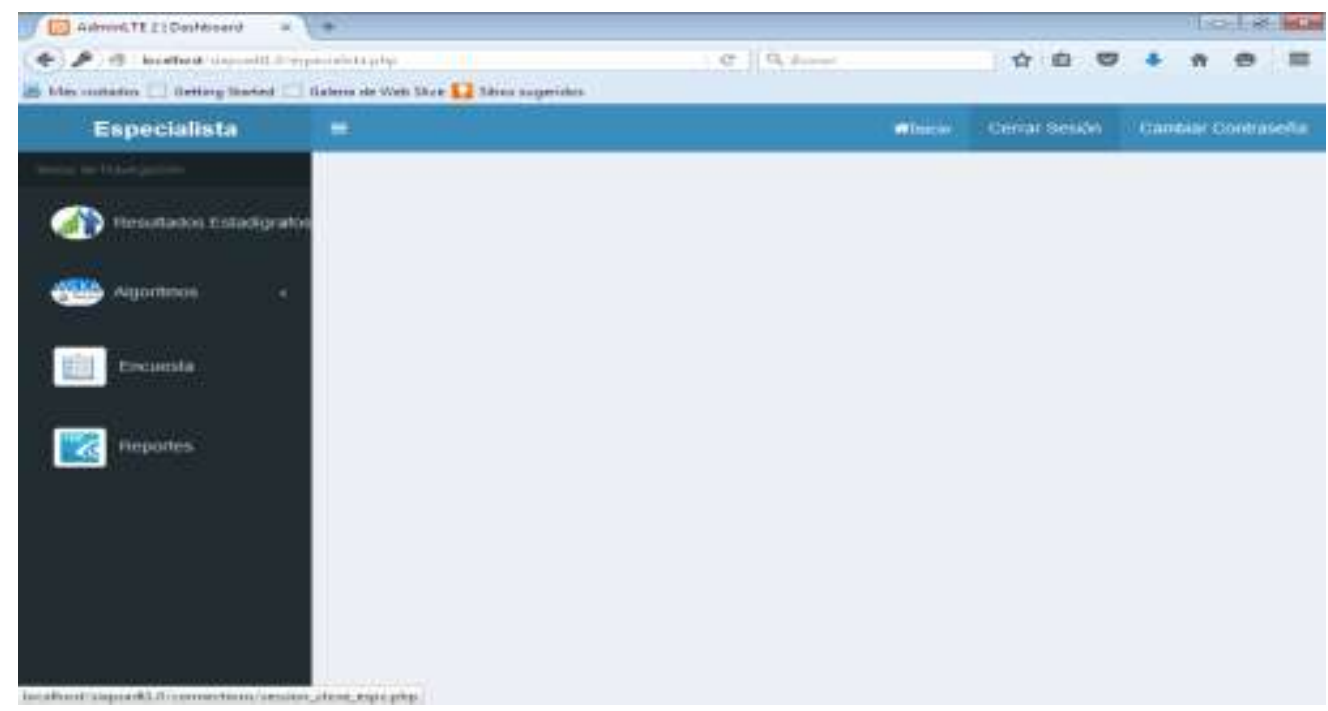

Figura 2: Interfaz del usuario Especialista.

Fuente: Elaboración propia.

En la figura 3 se muestra la salida donde el Especialista escoge la opción Gráfica Pastel y analiza los resultados pertinentes que visualizan la cantidad de encuestados por preguntas que pertenecen a cada grupo.

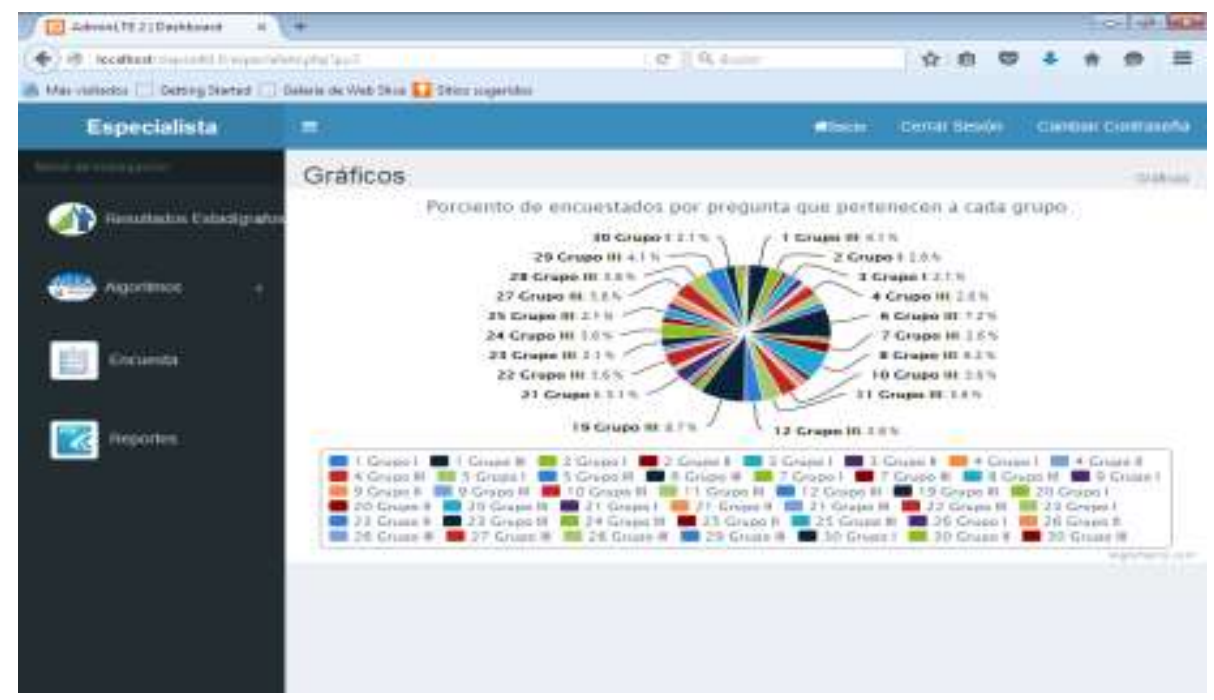

Figura 3: Interfaz de gráfico de pastel para la clasificación de la percepción por grupo.

Fuente: Elaboración propia. 
La figura 4 muestra la salida donde el Especialista escoge la opción Algoritmo y da la salida del algoritmo ID3, proporcionando al especialista conocer los parámetros que se tienen en cuenta durante el proceso de clasificación como son los casos correctamente clasificados, el índice estadístico Kappa que refleja la coorcondancia entre los encuestados, se interpreta que hay un acuerdo máximo entre todos (1) si no (0) que no hay.

La matriz de Confusión facilitara al especialista comprobar o por lo menos obtener una probabilidad del conjunto de instancias clasificadas según las respuestas de los encuestados en cada uno de los grupos de la escala de percepción por ejemplo la cantidad de instancias que se encuentran dentro de la percepción adecuada del peligro y las maneras de enfrentar los deslizamientos de tierra que sería el Grupo I, el conjunto de las respuestas que se encuentran dentro de la percepción cercana a la realidad pero insuficiente que sería el Grupo II: , y los que tienen una percepción errónea o nula del peligro y las maneras de enfrentarlo que sería el Grupo III.

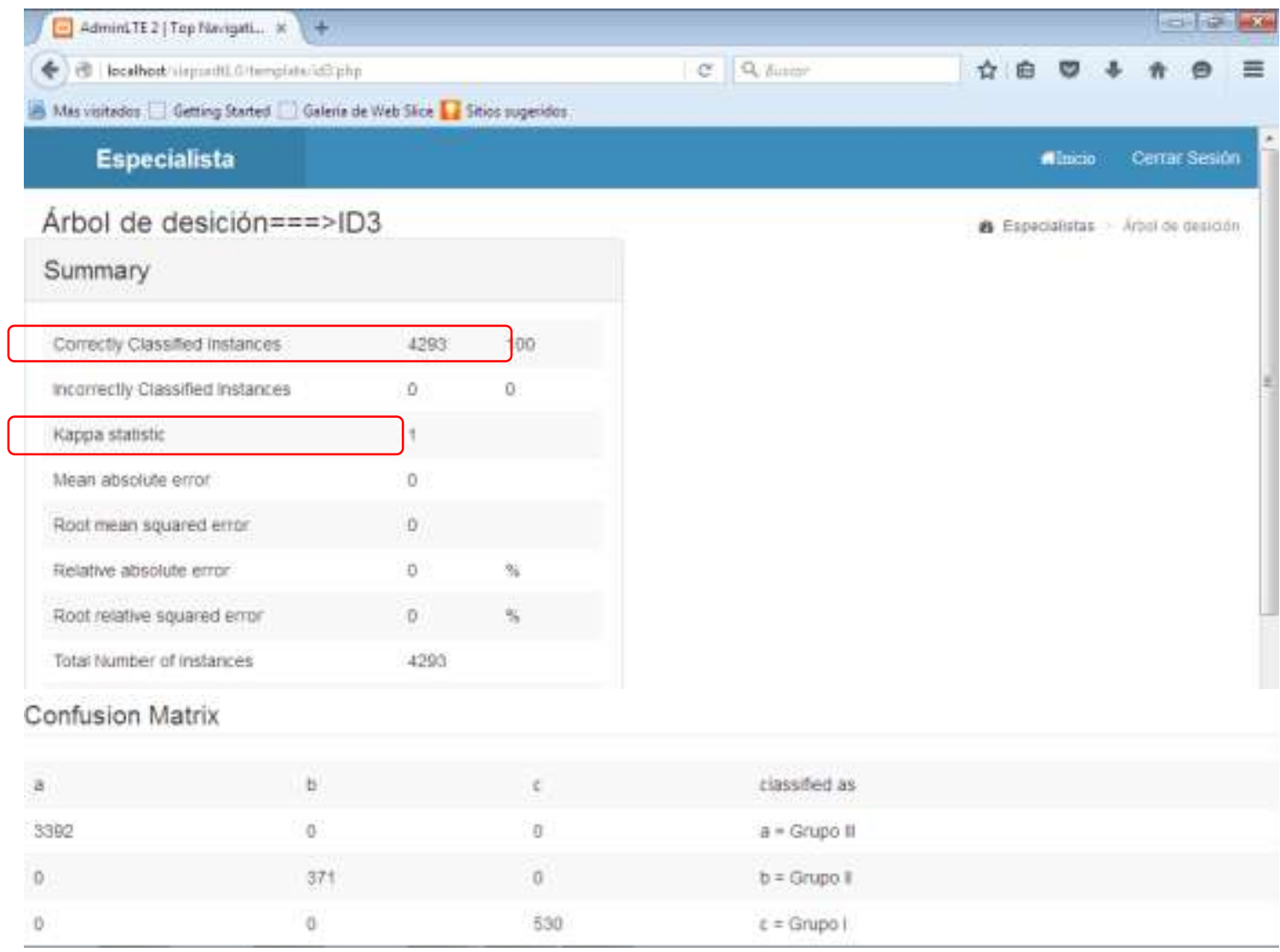

Figura 4: Árbol de decisión.

Fuente: Elaboración propia 


\section{Conclusiones}

En la investigación se arriba a las siguientes conclusiones:

- Consultaron los referentes teóricos sobre la investigación a realizar, enfocándose en la teoría referente a los sistemas informáticos relacionados con las percepciones socioambiental propiciando un mejor entendimiento del tema en cuestión.

- Diseñó el sistema para las percepciones socio-ambientales de los Deslizamientos de tierra utilizando las metodologías de ingeniería de software.

- Aplicaron los métodos matemáticos-computacionales y herramientas de desarrollo, enfocados hacia la elaboración del sistema informático.

- Implementó el sistema informático para el análisis de las percepciones socioambientales de los Deslizamientos de tierra y el mejoramiento de la toma de decisiones por parte de los actores locales e instituciones gubernamentales.

RECEIVED: April, 2017

REVISED: August, 2017

\section{Referencias bibliográficas}

[1] Calleja, N., Godefoy, E (2013). Principales resultados de los estudios de Percepción de los Peligros: Fuertes Vientos, Fuertes Lluvias y Penetración del mar, en Cuba.

[2] Carrasco, A. F. (2015). Módulo Gestión de Proyectos Internacionales para el Sistema de Información Gerencial del Centro Nacional de Sanidad Agropecuaria.

[3] Garreet, J; Sicilia, M; Rodríguez, D ; Rejas, R (2007).Comparación de diferentes algoritmos de clustering en la estimación de coste en el desarrollo de software.

[4] Gil, A; Lara, I (2016).Sistema Informático para el análisis de las percepciones socioambientales en los Deslizamientos de Tierra. Tesis presentada en opción al Título de Ingeniero en Informática. Universidad Agraria de la Habana. Provincia Mayabeque.120p.

[5] Guttman, E., Sánchez, C. Z., De Forero, A. C. \& Ramírez, J. C.(2004). Diseño de un sistema de indicadores socio ambientales para el Distrito Capital de Bogotá. CEPAL NACIONES UNIDAS. 
[6] Martínez, Q. (2009). Guía metodológica para desarrollar indicadores ambientales y de desarrollo sostenible en países de América Latina y el Caribe, CEPAL.

[7] Rodríguez, J. M. (2004). Geografía y cultura popular: reflexiones para el debate. Conferencia presentada en el IV Congreso Nacional de Geografía. Sociedad Cubana de Geografía.

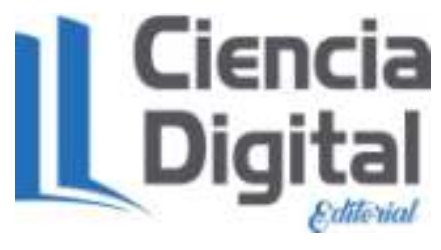

El artículo que se publica es de exclusiva responsabilidad de los autores y no necesariamente reflejan el pensamiento de la Revista Ciencia Digital.

El articulo queda en propiedad de la revista y, por tanto, su publicación parcial y/o total en otro medio tiene que ser autorizado por el director de la Revista Ciencia
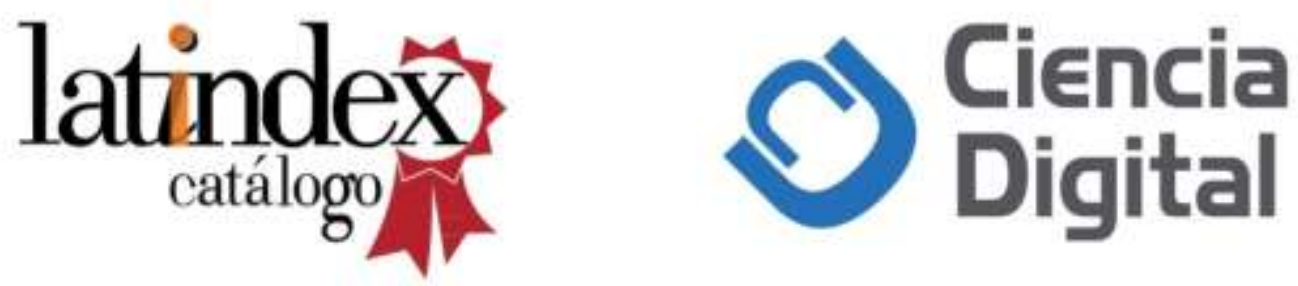